\title{
Quaderni
}

QUADERNI Communication, technologies, pouvoir

\section{La réflexivité communicationnelle, processus facilitant la construction d'une autorité dans les espaces médiatisés participatifs}

\section{Marion Rollandin}

\section{(2) OpenEdition Journals \\ Édition électronique \\ URL : http://journals.openedition.org/quaderni/1076 \\ DOI : 10.4000/quaderni. 1076 \\ ISSN : 2105-2956 \\ Éditeur \\ Les éditions de la Maison des sciences de l'Homme}

Édition imprimée

Date de publication : 5 mai 2017

Pagination : 53-61

Référence électronique

Marion Rollandin, «La réflexivité communicationnelle, processus facilitant la construction d'une autorité dans les espaces médiatisés participatifs », Quaderni [En ligne], 93 | Printemps 2017, mis en ligne le 05 mai 2019, consulté le 05 janvier 2020. URL : http://journals.openedition.org/quaderni/1076 ; DOI : 10.4000/quaderni.1076 


\section{$D$ ossier}

\section{La réflexivité communicationnelle, processus facilitant la construction d'une autorité dans les espaces médiatisés participatifs}

\author{
Marion \\ Rollandin
}

GRIPIC/Celsa Paris-Sorbonne
Les dispositifs médiatisés informatisés permettent l'interaction avec d'autres usagers. En offrant des espaces d'énonciation en ligne, ils sont porteurs d'une promesse d'opportunité de communication à tout moment grâce au grand nombre potentiel d'interlocuteurs et aux outils de communication utilisables quand on le désire. Or, ces opportunités s'avèrent difficiles à saisir en raison des spécificités de la communication médiatisée : les corps des utilisateurs restant dans l'ombre rendent l'amorce d'interaction difficile. Ne pas savoir qui est la personne derrière l'écran, ne pas voir ses réactions et ne pas avoir à soutenir son regard facilite le refus d'un échange ou facilite sa rupture. Cela est accentué dans les dispositifs qui permettent l'usage d'un pseudonyme, protégeant ainsi l'identité sociale, ce qui concourt à complexifier le développement d'une sociabilité sur le long terme. Dans ce contexte, mener à bien un projet de construction d'une autorité devient difficile. L'autorité est ici envisagée comme un processus d'autorisation personnelle et collective permettant la légitimation et la qualification dans un domaine d'expertise et de savoirs. En effet, comment être reconnu comme détenteur d'un savoir si les autres usagers refusent les situations d'échange ou si celles-ci ne durent pas suffisamment longtemps pour en faire état? Il convient alors pour chacun de déterminer les éléments du dispositif qui peuvent aider à se légitimer en tant qu'expert dans un domaine.

Dans la perspective de saisir les conditions de construction de l'autorité, nous avons étudié deux dispositifs utilisés par les sujets sur leur temps libre : le jeu multijoueur League of Legends ${ }^{1}$ et le site de discussion féminin Confidentielles ${ }^{2}$. Le cadre théorique mobilisé se compose de travaux 
dédiés aux médias informatisés et d'adaptations de théories pensées pour l'analyse des interactions en face à face (rôle, éthos...). Les deux terrains ont été investis par le biais d'une méthodologie ethnosémiotique ${ }^{3}$ où fonctionnent en symbiose une analyse sémio-pragmatique des dispositifs visant à décrire les situations de communication créées et les possibilités d'action auxquelles sont confrontés les usagers, et une analyse interactionnelle de terrain sur un temps long qui a permis de côtoyer les usagers et de les solliciter dans le cadre d'entretiens en autoconfrontation.

Nous avançons l'hypothèse que la réflexivité communicationnelle induite par la pratique d'un de ces deux dispositifs ${ }^{4}$ aide les sujets à mener à bien leur projet de construction de l'autorité. La réflexivité communicationnelle est un processus actif sur plusieurs niveaux : la mise à distance des expériences vécues afin de les comparer à d'autres (médiatisées ou non), la mise en perspective des outils offerts par les dispositifs par rapport à ce que l'on a l'habitude de faire dans des situations de communication en face à face, et le décryptage des traces laissées par les autres usagers et de leur propre réflexivité transparaissant dans les échanges. Ces opérations réflexives participent petit à petit à la construction d'un savoir sur la communication médiatisée et sur la gestion de la sociabilité en ligne. Ce processus se déroule dans des dispositifs proposant des expériences qui se vivent généralement sur un temps long, ce qui permet également la construction d'un savoir sur l'activité elle-même. Ces différents savoirs sont développés par les usagers à force de pratique, et peuvent faire l'objet d'un projet de construction de l'autorité. Pour cela, il est essentiel d'obtenir le droit à la parole avant même d'entreprendre d'être reconnu comme détenteur d'une certaine expertise. Dans un premier temps, nous porterons notre attention sur la nécessité pour chacun d'acquérir le droit à la parole. Dans un second temps, nous analyserons les tactiques permettant la qualification en tant qu'expert de l'activité grâce aux éléments présents dans le dispositif.

\section{Être légitime à communiquer dans les espaces médiatisés informatisés}

On observe que les usagers se heurtent dès leurs débuts à des difficultés d'amorce d'interaction. S'inscrire sur LoL ou Confi permet d'exister " informatiquement », mais n'est pas suffisant pour acquérir une existence sociale. Ainsi, inscrire un message à l'attention d'un interlocuteur n'implique pas nécessairement une réponse de sa part, celle-ci dépendant de son bon vouloir. Le dispositif médiatisé ne dispose pas d'outils pour l'obliger à considérer une sollicitation. Chacun doit donc déterminer ce qui motive l'obtention du droit de parole. Deux éléments fondamentaux ont été mis en exergue.

Le premier élément correspond à l'aptitude que doit montrer chaque usager à cerner les attentes de l'institution et des autres usagers, en décryptant le cadre de l'activité et en adoptant un rôle cohérent avec celui-ci. Si la compréhension des situations de communication en face à face s'effectue grâce aux lieux dans lesquels on se trouve, à l'identité sociale et au comportement des autres personnes impliquées, sur un dispositif médiatisé, un flottement s'impose. La non-visibilité de l'interlocuteur cache une partie des éléments habituellement décryptés pour définir comment se comporter. Par des opérations réflexives, les usagers vont 
définir d'autres éléments à interpréter pour les aider à comprendre ce qui se joue dans l'activité. La situation de communication prend place dans un espace numérique conçu par une institution, regorgeant de signes à interpréter. Chaque usager qui s'engage dans une activité médiatisée se confronte ainsi à des outils du dispositif et des textes institutionnels présentant l'univers et ses règles, marqués par une "ostension d'une intention communicationnelle lisible ${ }^{5}$ " c'est-à-dire qu'ils sont porteurs d'une représentation de ce que doit être la communication telle qu'envisagée par l'institution. Ces éléments inscrits à l'écran peuvent être interprétés afin de définir le cadre primaire de l'expérience. C'est "un cadre qui nous permet, dans une situation donnée, d'accorder du sens à tel ou tel de ses aspects, lequel autrement serait dépourvu de signification. ${ }^{6}$ " Par exemple, l'analyse du site officiel de LoL décrit le jeu comme un cadre de performance ludique collaborative ${ }^{7}$. Sur Confi, l'analyse de la page d'accueil montre que l'activité est définie comme un cadre de discussion genrée et d'information/consommation ${ }^{8}$. La perception du cadre primaire de l'activité révèle des schèmes qui correspondent " à une gamme d'actions et de comportements attendus par l'institution conceptrice, qu'elle souhaiterait voir adoptés par chaque usager. ${ }^{9}$ " Ces schèmes définissent le rôle de l'usager-modèle, usager fictif pour qui l'institution a conçu son dispositif. On distingue «le rôle-modèle pensé par l'institution, dont les contours peuvent être aisément perçus, du rôle endossé, qui n'est pas artificiel et résulte d'une adaptation interprétative de cette ligne directrice. ${ }^{10} \gg$ Chacun interprète et adopte un rôle lié au rôle modèle en fonction de ses expériences passées et de ses aspirations. Étant donné que les informations permettant d'interpréter cette ligne directrice recommandée sont visibles par tous les usagers, elles concourent donc à former chez chacun des attentes quant au rôle suivi par les autres. Dès lors, une comparaison s'engage entre le rôle modèle et le comportement adopté par les autres participants ${ }^{11}$. Elle constitue un indicateur sur leur capacité à savoir décrypter les enjeux de l'activité médiatisée et à accepter de se conformer à un cadre de l'activité donné. Cela permet à de potentiels interlocuteurs de faire des conjectures sur le déroulement de l'échange. Endosser un rôle éloigné du rôle-modèle introduit le risque pour les interlocuteurs de ne pas savoir comment se comporter en retour provoquant des réticences à s'engager dans une interaction. Une telle personne sera catégorisée comme " déviante ${ }^{12}$ ", ayant un comportement contraire aux attentes, risquant alors l'exclusion et la non-considération. On relève par exemple sur Confi des messages d'une utilisatrice incohérents avec les fils de discussion dans lesquels ils s'intègrent, sans qu'aucune remarque ne lui soit faite ${ }^{13}$. Les autres usagers nous confièrent qu'ils ne comprenaient pas ses motivations. N'arrivant pas à définir dans quel cadre de l'activité elle évoluait, ils avaient décidé de ne pas prêter attention à ses messages, refusant de la considérer comme un usager, étant donné qu'elle ne faisait pas l'effort d'inscrire des propos compréhensibles par tous, composante principale du rôle de « Confinaute».

Le second élément donnant droit à la parole correspond à la nécessité de témoigner à ses partenaires temporaires une certaine considération, tout en gérant les éventuelles difficultés liées à la situation médiatisée. Une fois le droit à la parole obtenu, il doit être entretenu en montrant 
du respect pour la situation de communication établie et pour ses participants. Un des signes faisant sens comme témoignage de respect est le maintien d'un engagement dans l'activité. On considère l'engagement comme l'attention, le degré de considération accordée à l'activité et à ses participants. C'est une « obligation socialisante. ${ }^{14}$ " Ainsi, à travers les traces laissées dans le dispositif au moment de l'activité, les usagers doivent justifier d'une attention continue suffisante au bon déroulement de l'activité médiatisée. Il convient par exemple de gérer son personnage sur LoL durant la partie de jeu pour aider son équipe à remporter la victoire et de répondre sur Confi de manière régulière pour qu'une sensation de dialogue soit perçue. L'engagement est une composante implicite du rôle général de « participant à l'interaction » issue des situations de communication en face à face : il est mal vu de ne pas s'engager totalement dans l'interaction avec une personne, en faisant en sa présence une autre activité l'excluant. En effet Goffman indique que « les acteurs donnent souvent l'impression que leur rôle actuel est leur seul rôle, du moins, le plus important. ${ }^{15}$ » Le public d'une interaction attend donc que son interlocuteur se consacre entièrement à son rôle, c'est-à-dire s'engage à le maintenir pour mener à bien l'activité. Cela est aussi valable pour les situations de communication médiatisée. Sur Confi, les échanges étant asynchrones, il est possible de ne pas répondre immédiatement. Si l'attente dépasse deux jours, des messages des autres participants apparaissent, témoignant des doutes sur la considération que l'interlocuteur en cause accorde à l'échange en cours, comme «la concernée est disparue » ou "c'est dommage de lancer des sujets pour rien. ${ }^{16}$ " Sur LoL, les conséquences d'une baisse de l'engagement d'un joueur influent sur l'issue de la partie : mettre en suspens le contrôle de son personnage peut se solder par sa mort temporaire, avantageant l'équipe adverse. Si le joueur se désengage de la partie pendant une durée trop longue, celle-ci sera perdue, ses coéquipiers se retrouvant à se battre en sous-nombre. Étant considéré comme n'ayant pas tout mis en œuvre pour le bien du groupe, il ne sera pas réinvité par les autres joueurs. Les diminutions de l'attention accordée à l'activité exposent un usager à la perte de son statut de partenaire de l'activité et au risque d'exclusion. On s'attend donc à ce que les usagers fassent preuve d'un engagement total dans l'activité. Or, cet engagement est difficilement constant, car la communication médiatisée place les utilisateurs dans une superposition de scènes d'interaction, appelée « état de polytopie synchronique ${ }^{17} »:$ le corps de l'usager est engagé dans l'activité par ses actions sur le clavier, le regard et les processus cognitifs dirigés vers l'écran alors que le corps reste visible dans l'espace physique, disponible pour d'autres activités ou d'autres situations de communication avec une personne de l'entourage ou d'un autre dispositif médiatisé. Cet état nuit à la conservation d'un engagement total dans l'activité médiatisée, en augmentant les potentielles sollicitations extérieures qui ne sont pas vues/ perçues par les partenaires médiatisés. Ainsi les baisses de l'engagement non gérées risquent de faire perdre à l'usager en cause son droit à la parole et son statut de partenaire. Pour pallier ces risques d'exclusion, les sujets développent des tactiques. Sur Confi, de nombreux posts témoignent d'opérations de réparation et de justification. Les utilisatrices anticipent les obligations qui les amèneront à ne pas se connecter sur 
le forum, avertissant ainsi les autres, s'excusant par avance de ne pas pouvoir répondre. Sur LoL, certains joueurs prétextent très souvent avoir un problème technique alors qu'ils étaient en fait en train de faire une autre activité.

\section{Être reconnu comme expert, légitime à partager ses savoirs}

Par la récurrence de situations au cours de l'usage d'un dispositif médiatisé, les usagers développent également des connaissances constituant des savoirs sur l'activité, que ce soit des stratégies individuelles ou collectives sur LoL ou des avis justifiés en lien avec des sujets intimes sur Confi. Ils peuvent, à un stade avancé de la pratique, chercher à être reconnus comme ayant une certaine expérience, et tenter de faire état de leurs connaissances. Des opérations réflexives les amènent à analyser les éléments qui les aideront dans le projet de construction de cette autorité et ceux qui le desserviront. Ces éléments sont notamment des données chiffrées qui traduisent une intensité de pratique qu'on lie souvent à une meilleure connaissance de l'activité. Nous allons voir qu'ils n'ont pas la même signification dans les deux espaces étudiés.

D'une part, les données chiffrées sur LoL sont un gage d'autorité reconnu par les autres. Ces données résultent des actions des usagers. Ils laissent en effet des traces dans le dispositif, traces volontaires lorsqu'il s'agit d'inscrire des propos écrits comme le choix du pseudonyme ou bien involontaires lorsqu'il s'agit des enregistrements des actions effectuées sur le dispositif. Nous entendons les traces au sens de "productions présentes [...] comme des indices de pratiques qui ont lieu [...]. Mais pour qu'une représentation de pratique soit présente sur le site - et donc dans les termes considérés ici, que le texte porte trace d'un usage-il ne suffit pas que cette pratique ait lieu. Elle doit avoir fait l'objet d'un acte d'inscription, réalisé par son auteur ou par une autre personne, ou encore engendré par un dispositif d'enregistrement automatique. Ces procédures ont en commun de se matérialiser, pour un public, par un objet visuel prenant la forme d'un tracé interprétable dans le cadre d'une culture écrite donnée. ${ }^{18}$ " Une partie de ces traces participent à la construction d'un profil d'utilisateur, visible par tous. Elles peuvent être interprétées comme signes d'une expertise par les usagers " aguerris », c'est-à-dire ceux familiers avec la culture de l'activité proposée. Les nouveaux usagers n'ont pas conscience de cette connotation, et prennent petit à petit conscience de l'importance qu'elle aura sur leur image. La première connotation qu'ils découvrent est celle du niveau. En effet, ils débutent le jeu avec un compte de jeu de niveau 1 , qu'ils feront évoluer au niveau 30 à force de pratique. Cela dure plusieurs mois, période au cours de laquelle ils se familiarisent avec les outils du dispositif et les règles explicites et implicites. Lorsque deux joueurs n'ont pas le même niveau de compte, celui qui a le plus élevé se considère comme plus expérimenté et n'accorde que très peu d'importance à l'avis émis par celui qui est considéré comme moins expérimenté. Nous avons nous-mêmes vécu cette différence : la joueuse de niveau 30 avec qui nous évoluions alors que nous étions au niveau 9 émettait des jugements très critiques vis-à-vis des choix stratégiques que nous proposions, même si ceux-ci guidaient notre équipe vers la victoire. Toutefois, au niveau 30 , les joueurs se heurtent à des difficultés similaires 
qui prennent la forme d'un nouvel indicateur : la place dans le classement. Le résultat de leurs parties les intègre dans un classement comportant des paliers. La place dans le classement visible sur le profil peut nuire au projet de construction d'une autorité. Des joueurs, identifiables comme étant dans le bas du classement, nous ont confié que cela était extrêmement stigmatisant, les autres joueurs les considérant comme ayant un mauvais niveau allant même jusqu'à leur refuser le droit à la parole. Cela incita certains à prêter leur compte de jeu à d'autres joueurs pour qu'ils les aident à progresser dans le classement. Un joueur ayant eu recours à ce stratagème interdit par l'éditeur de jeu m'indiqua qu'avec un classement « argent », les groupes de jeu acceptaient désormais de considérer ses conseils en termes de stratégie.

D'autre part, les données chiffrées sur Confi participent à la reconnaissance par l'institution d'une forme de savoir sur l'activité, permettant à certaines utilisatrices d'obtenir des statuts spéciaux se matérialisant par des signes distinctifs. Si les traces de participation font sens pour les usagers, elles font également sens pour l'institution qui s'occupe du dispositif de Confi, qui les place comme condition essentielle permettant d'acquérir deux statuts particuliers. Le premier statut est celui de «Confisage», que nous avons identifié comme équivalent à une « police de proximité » dont l'objectif est de surveiller le bon déroulement des interactions, et de signaler aux administrateurs tout débordement qu'elles n'arriveraient pas à contrôler. Ce statut peut être demandé à condition d'être membre fréquentant le site depuis plus de 6 mois et de ne pas avoir reçu d'avertissement de la part de l'administra- tion du site. Une utilisatrice ayant demandé et obtenu ce statut arbore fièrement sur son profil une icône représentant un ange, symbolisant ainsi leur droit de diffuser la parole de l'institution. Elle explique : «je n'aime pas quand des personnes viennent pour mettre le bazar. Non pas que je me considère comme la justicière de Confidentielles, mais au moins ceux qui viennent juste pour ça, ils savent que je suis en droit de les remettre à leur place ». Ainsi, l'attribution de ce signe distinctif signifie pour les autres qu'elle est légitime à gérer les débordements, qu'elle détient des connaissances la rendant apte à juger les « bonnes pratiques de la communication », articulant une connaissance des normes et règles explicites données par l'institution, avec des règles implicites issues des situations de communication en face à face, comme les règles de bienséance. Le second statut relevé sur le site est celui de « Confitoque ». Il « regroupe des utilisatrices ayant une expertise dans le domaine culinaire, capable de transmettre aux autres leur savoir par le biais d'une contribution [...] qui sont des fiches de recettes de cuisine..$^{19} \gg \mathrm{Si}$ les conditions d'obtention de ce statut n'ont pas toujours été les mêmes, au moment de notre étude, il fallait avoir partagé un certain nombre de recettes avant de faire une demande auprès du service client du site. Les traces d'usages devaient ensuite témoigner d'un fort investissement afin de conserver ce statut. Une utilisatrice commentait « il faut que je reste active si je veux garder ce statut». Concrètement, il lui était demandé de poster deux recettes de cuisine par mois et d'écrire au moins deux messages dans le forum « Cuisine».

Dans les deux dispositifs étudiés, des traces résultant d'un usage - qu'elles soient automa- 
tisées comme les indicateurs de niveau sur LoL ou attribuées comme les statuts sur Confi - sont interprétées par les autres usagers comme un signe d'autorité, un gage témoignant d'une certaine expertise.

Pour conclure, nous avons montré que la réflexivité communicationnelle était un processus facilitant la construction de l'autorité dans les espaces médiatisés participatifs. Elle pousse les usagers à comparer les situations de communication médiatisée à celles en face à face, cherchant à déterminer les éléments qui les aideront à se comporter au cours de l'activité. Elle participe tout d'abord à la définition des éléments qui permettent aux usagers de se faire admettre comme un partenaire de l'échange qui a le droit à la parole. Ils ajustent ainsi leur comportement aux attentes de l'institution et des autres usagers et aux difficultés liées à la situation de communication médiatisée (non-visibilité des corps et état de polytopie synchronique). Elle facilite aussi pour les usagers désireux de s'engager dans un projet d'autorité l'estimation de l'impact des traces d'usages laissées dans le dispositif sur leur éthos médiatisé. Cela leur permet d'élaborer des tactiques ou une ligne d'action qui leur faciliterait la reconnaissance de leur expertise. En guise d'ouverture, nous attirons l'attention sur un constat : des dynamiques de groupe ont été observées, dans lesquelles les usagers défendent le savoir dont fait état un des leurs, face à une personne extérieure au groupe qui tenterait de remettre en cause cette expertise. L'appartenance à un groupe favorise le développement d'autorités individuelles qui deviennent alors des autorités collectives.

\section{$R \cdot \hat{E} \cdot F \cdot \dot{E} \cdot R \cdot E \cdot N \cdot C \cdot E \cdot S$}

BECKER Howard, Outsider : étude de sociologie de la déviance, Paris, A.-M. Métailié, 1985. GALINON-MÉLÉNEC Béatrice (dir.), L'homme trace-Perspectives anthropologiques des traces contemporaines, Paris, CNRS Éditions, 2011. GOFFMAN Erving, La mise en scène de la vie quotidienne. Tome 1 : La présentation de soi. Paris, Les Éditions de Minuit, 1973.

GOFFMAN Erving, Les cadres de l'expérience, Paris, les Éditions de Minuit, 1991.

JEANNERET Yves, Penser la trivialité volume 1 : la vie triviale des êtres culturels, Paris, Lavoisier, 2008.

ROLLANDIN Marion, La réflexivité communicationnelle induite par les échanges en ligne: pratique, médiation et médiatisation, vers une posture d'ethnologue-amateur, Th. Doct : Sciences de l'Information et de la Communication, sous la direction d'Yves Jeanneret et de Valérie Jeanne-Perrier, CELSA - Université Paris-Sorbonne, 2015.

SOUCHIER Emmanuel (dir.), JEANNERET Yves, LE MAREC Joëlle, Lire, écrire, récrire. Paris, Bibliothèque publique d'information, 2003. 
$\mathrm{N} \cdot \mathrm{O} \cdot \mathrm{T} \cdot \mathrm{E} \cdot \mathrm{S}$

1. League of Legends (abrégé LoL) est une arène de bataille en ligne multijoueur. Deux équipes de cinq joueurs s'affrontent par le biais de personnages prédéfinis au cours de parties de 45 minutes. Les espaces d'énonciation écrits sont accessibles au cours des parties sous la forme d'un tchat pour les membres des deux équipes ou sur le temps d'attente entre deux parties avec des joueurs dont on connaît le pseudonyme prenant alors la forme d'une fenêtre de messagerie privée.

2. Confidentielles (abrégé Confi) est un site de discussion généraliste en ligne dédié aux femmes. Les discussions écrites se font sur des forums thématiques (autour de préoccupations féminines) publics, visibles par tous. Une messagerie permet aussi de correspondre en privé. Dans cet article, nous tenons compte de la version du site telle qu'elle était avant avril 2014, les thématiques du site ayant été ensuite réorientées exclusivement sur la beauté.

3. Le Marec et Babou in Souchier Emmanuel (dir.), Jeanneret Yves, Le Marec Joëlle, Lire, écrire, récrire, Paris, Bibliothèque publique d'information, 2003, p. 237.

4. Nos travaux de thèse ont montré que leur pratique permettait le développement d'un processus réflexif accru à l'égard des situations de communication en ligne. (Rollandin, 2015).

5. Jeanneret Yves, Penser la trivialité - volume 1 : la vie triviale des êtres culturels, Paris, Lavoisier, 2008, p. 61. 6. Goffman Erving, Les cadres de l'expérience, Paris, les Éditions de Minuit, 1991, p. 30.

7. Rollandin Marion, La réflexivité communicationnelle induite par les échanges en ligne : pratique, médiation et médiatisation, vers une posture d'ethnologue-amateur, Th. Doct : Sciences de l'Information et de la Communication, sous la direction d'Yves Jeanneret et de Valérie Jeanne-Perrier, CELSA - Université Paris-Sorbonne, 2015, pp. 126-129.

8. Ibid., pp. 131-133.

9. Ibid., p. 141.

10. Ibid., p. 140.

11. Cette comparaison est possible par l'interprétation de la forme de l'amorce de l'interaction et des premiers échanges qui vont suivre.

12. Becker Howard, Outsider : étude de sociologie de la déviance, Paris : A.-M. Métailié, 1985.

13. Rollandin Marion, op. cit., p. 143.

14. Goffman, op. cit., 1991, p. 339.

15. Goffman Erving, La mise en scène de la vie quotidienne. Tome 1 : La présentation de soi. Paris : Les Éditions de Minuit, 1973, p. 191.

16. Rollandin, op. cit., p. 358.

17. Ibid., p. 166.

18. Jeanneret in Galinon-Mélénec Béatrice (dir.), L'homme trace - Perspectives anthropologiques des traces contemporaines, Paris, CNRS Éditions, 2011, p. 72 .

19. Marion Rollandin, op. cit., p. 419. 
$R \cdot E \cdot S \cdot U \cdot M \cdot E ́$

L'objet de cet article est de saisir les conditions de construction de l'autorité dans des espaces médiatisés participatifs tels que les jeux multijoueurs en ligne ou les forums de discussion. Cette construction de l'autorité s'avère complexe dans ces espaces. En effet, ils présentent un paradoxe : ils donnent de nombreuses occasions d'interaction tout en facilitant les refus d'amorce des échanges et l'exclusion. Nous montrons que chaque individu qui s'engage dans un projet de construction de l'autorité développe un processus appelé la réflexivité communicationnelle. D'une part, ce processus permet aux usagers de déterminer quel comportement adopter pour augmenter leurs chances d'être acceptés par les autres usagers en tant que partenaires de l'échange. D'autre part, il favorise l'élaboration de tactiques facilitant la reconnaissance de l'expertise chez chaque personne désirant partager un savoir sur l'activité ou la communication.

\begin{abstract}
The purpose of this article is to define the conditions of authority construction in participatory mediatized spaces, such as online multiplayer games or discussion forums. This construction of authority is, as shown, complex in these areas. Indeed, they present a paradox : they provide many opportunities of interaction while facilitating refusal to start exchanges, and exclusion as well. We show that the participants develop a process called communicational reflexivity, whenever they get involved in any authority construction project. On the one hand, this process is what allows them to determine what behavior they should adopt in order to increase their chances of being accepted by other people as exchange partners. On the other hand, it promotes the development of tactics facilitating the recognition of
\end{abstract}

the expertise for each one who wants to share their knowledge about activity or communication. 
